\title{
O Uso de um Aplicativo como Ferramenta para o Ensino de Conceitos de Climatologia em Escola Pública do Ceará
}

\author{
José Bruno Marques Fernandes ${ }^{1}$, Emerson Mariano da Silva ${ }^{1}$ (D), José Valdemir dos Reis Júnior ${ }^{2}$ \\ ${ }^{1}$ Universidade Estadual do Ceará, Fortaleza, CE, Brasil. \\ ${ }^{2}$ Universidade Federal do Piauí, Teresina, PI, Brasil.
}

Recebido em: 9 de Abril de 2020 - Aceito em: 7 de Maio de 2020

\begin{abstract}
Resumo
Em meio ao mundo digital do século XXI, trabalhar com educação tornou-se desafio, sobretudo para os professores, que precisam estar atentos às mudanças provocadas pelo avanço das tecnologias no ensino. Assim, conteúdos que antes pareciam ser de difícil entendimento por parte dos discentes podem agora ser mais bem compreendidos com o auxílio de novas ferramentas educacionais, a exemplo dos aplicativos de celular voltados para o ensino nas mais diversas áreas do conhecimento. Pensando em facilitar o ensino e a aprendizagem de conceitos ligados ao clima e aos principais sistemas meteorológicos responsáveis pelas chuvas no estado do Ceará, foi desenvolvido o aplicativo intitulado "ClimaCE", que tem o objetivo de ser mais uma ferramenta disponível ao docente e aos estudantes do estado do Ceará, sobretudo da rede estadual de ensino. O aplicativo pode ser utilizado em sala de aula por professores da disciplina de Geografia e de eletivas de áreas afins. Conta, inclusive com um Quiz relacionado ao conteúdo, e busca despertar nos estudantes cearenses o interesse pela Climatologia. Ademais, o aplicativo também pode interessar outros grupos, fora da dinâmica escolar, que venham a ter acesso a ele através de download na loja de aplicativos.
\end{abstract}

Palavras-chave: aplicativo, educação, clima, sistemas meteorológicos.

\section{The Use of an Application as a Tool for Teaching Climatology Concepts in a Public School in Ceará}

\begin{abstract}
In the digital world of the 21 st century, to work with education has become a challenge, especially for teachers, who need to be aware of the changes due to the advancement of technologies in teaching. Thus, some contents that previously seemed to be difficult for leaners to understand can now be better understood with the help of new educational tools, such as teaching mobile applications in various areas of knowledge. With the propose to make easier the teaching and learning of concepts related to climate and the main meteorological systems responsible for rainfall in the state of Ceará, the application entitle "ClimaCE" was developed, which aims to be another tool available to teachers and students, mainly the ones who belong to the state school system. Geography and related areas teachers can use the app in the classroom. It also includes a quiz related to the content, and it aims to awake Ceará students' attention to Climatology studies. In addition, the app may also interest other non-school groups who may have access to it by downloading it from the app store.
\end{abstract}

Keywords: app, education, climate, meteorological systems.

\section{Introdução}

Há muito tempo o homem procura aprimorar os conhecimentos ligados ao clima e tenta, através de vários métodos, prever se vai chover ou não. A busca por essa resposta une de acordo com Folhes e Nelson (2007) conhecimento popular e ciência.

Com o aprimoramento das tecnologias na área da computação e da meteorologia, a previsão do tempo atin-

Autor de correspondência: José Bruno Marques Fernandes, jbrunomarquesf@gmail.com. 
giu maior grau de precisão. Os profissionais que trabalham nessa área passaram a ter disponíveis, de acordo com Vianello e Alves (2000), imagens de satélite, as quais, aliadas a modernos equipamentos e modelos de simulação físicomatemáticos, são capazes de informar, com horas de antecedência, a previsão do tempo em diferentes cidades do mundo.

Desde sua fase nômade, o homem altera de alguma forma a natureza, gerando impactos no meio ambiente. Com o advento da Primeira Revolução Industrial, ocorrida no século XVIII na Inglaterra, e o aumento no uso de combustíveis fósseis, as concentrações de Gases de Efeito Estufa (GEE) na atmosfera se intensificaram, contribuindo para a ocorrência do aquecimento global e de mudanças climáticas, as quais, segundo Blank (2015) "talvez sejam o maior desafio imposto à humanidade desde o surgimento do mundo moderno".

O aumento das temperaturas está provocando alterações climáticas que já começam a ser perceptíveis em várias regiões do planeta. No Brasil, estudos do Painel Brasileiro de Mudanças Climáticas - PBMC (2019) indicam que uma das áreas que serão mais atingidas pelas mudanças climáticas será a região Semiárida.

Diante desse quadro de mudanças climáticas, estabelecer estratégias para que as pessoas possam aprender a conviver com a seca na região Semiárida do Brasil é de fundamental importância para que se evite uma migração em massa de pessoas, como aconteceu no passado. No caso do Ceará, estado do Nordeste inserido no polígono das secas, relatos desse período podem ser encontrados em obras literárias como "O Quinze", de Rachel de Queiroz (98 $\left.{ }^{\mathrm{a}} \mathrm{Ed}, 2015\right)$.

O estudo da situação climática do estado do Ceará deve começar na escola, cabendo ao professor de Geografia e a outros profissionais de áreas afins explicarem aos seus alunos, ao longo dos assuntos relacionados à abordagem desse conteúdo, como por exemplo, conceitos ligados ao clima Semiárido e aos sistemas meteorológicos que influenciam nas precipitações do estado. Porém, esse conteúdo é visto em sala de aula sem um maior aprofundamento.

Muitas vezes, por falta de materiais didáticos específicos para que o professor possa explicar melhor aos alunos sobre a situação climática do estado do Ceará, os estudantes, sobretudo os de Fortaleza, que não convivem com a realidade da seca de perto diariamente acabam não compreendendo conceitos ligados ao clima Semiárido, e a importância de diferentes sistemas meteorológicos, como a Zona de Convergência Intertropical (ZCIT), para as precipitações e recarga dos reservatórios cearenses.

Buscando melhorar a compreensão dos estudantes acerca de conceitos ligados ao clima Semiárido - típico do interior do Nordeste e predominante no interior do estado do Ceará - bem como de informar sobre os sistemas meteorológicos atuantes no estado durante os períodos de
Pré-estação, Período chuvoso e Pós-estação, foi desenvolvido o aplicativo intitulado "ClimaCE".

O aplicativo utiliza informações disponíveis em sites de órgãos de monitoramento hídrico do estado, tais como o site da Fundação Cearense de Meteorologia e Recursos Hídricos (FUNCEME) e do portal hidrológico do Ceará.

Assim, os estudantes podem encontrar de maneira mais rápida e fácil, reunidas no aplicativo, informações sobre o clima Semiárido e os principais sistemas meteorológicos atuantes no estado do Ceará, além de um Quiz com dez perguntas relacionadas ao conteúdo disponível no aplicativo.

\section{Material e Métodos}

Para a construção do aplicativo intitulado "ClimaCE", inicialmente foi realizada uma revisão de literatura sobre a ocorrência dos eventos de seca no Semiárido brasileiro, em especial no estado do Ceará. Na pesquisa bibliográfica, foram apresentados os principais sistemas meteorológicos que causam chuva no Nordeste do Brasil e, em particular, no norte da região, e que, portanto, influenciam diretamente nas precipitações dos municípios cearenses.

Além disso, foram repassadas informações sobre os fenômenos El Niño, La Niña e Dipolo do Atlântico, também chamado de Gradiente Meridional de anomalias de TSM no Atlântico Tropical, que podem interferir no regime de chuvas do estado. Algumas curiosidades, tais como a diferença entre "inverno" e período chuvoso, tema que causa muita confusão entre os alunos, e dados sobre o açude Castanhão, considerado o maior reservatório da América Latina, também foram incluídos no aplicativo.

Ao acessar a ferramenta, conforme indicado nas Figs. 1 e 2, o usuário (alunos e/ou público em geral) visualiza uma tela principal, com as opções que podem ser selecionadas, na seguinte ordem: Informações Gerais sobre o estado do Ceará (1), Tempo Meteorológico (2), Clima (3), Principais sistemas meteorológicos que provocam chuva no estado do Ceará (4), Fenômenos que interferem nas chuvas no estado do Ceará (5), Você Sabia? (6), Teste seus conhecimentos (7) e Sugestões de sites (8).

Ao selecionar na tela inicial a opção 1 - Informações Gerais sobre o Ceará, o estudante e/ou usuário é direcionado para uma aba informando o clima típico do estado e a quantidade de municípios cearenses inseridos no Semiárido, conforme pode ser observado através da Fig. 3.

As abas 2 e 3 do aplicativo ClimaCE, intituladas "Tempo Meteorológico" "Clima" buscam explicar as diferenças existentes entre esses dois conceitos, apresentando inclusive exemplos de fatores climáticos, como mostram as Figs. 4, 5 e 6.

A aba 4 - Principais sistemas meteorológicos que provocam chuva no estado do Ceará mostram informações 


\section{ClimaGE}

\section{$4 \quad 0 \quad 0$}

Figura 1 - Tela inicial do Aplicativo ClimaCE.

\section{Clima CE}

\author{
1. Informações Gerais sobre o Ceará \\ 2. Tempo meteorológico \\ 3. Clima \\ 4. Principais sistemas meteorológicos que \\ provocam chuva no estado do Ceará \\ 5. Fenômenos que interferem nas chuvas no \\ estado do Ceará \\ 6. Você Sabia? \\ 7. Teste seus conhecimentos \\ 8. Sugestões de sites
}

\section{( $\square$ \\ 4}

Figura 2 - Opções da Tela inicial do Aplicativo ClimaCE. $\leftarrow \quad$ ClimaCE

\section{Informações Gerais sobre o Ceará}

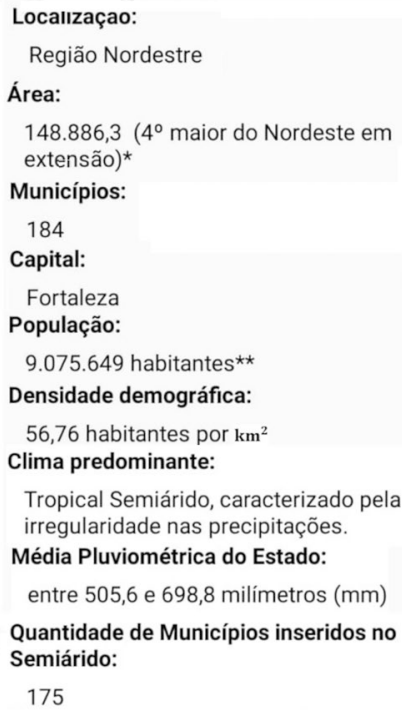

Quantidade de Municípios inseridos no Semiárido:

175

Figura 3 - Informações Gerais sobre o Ceará

\section{Tempo Meteorológico}

Por Tempo meteorológico, pode-se entender o estado momentâneo da atmosfera de um determinado lugar.

O tempo pode se apresentar de diferentes maneiras, como por exemplo:

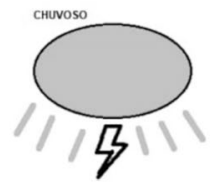

Chuvoso

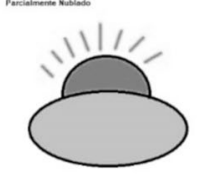

Parcialmente Nublado

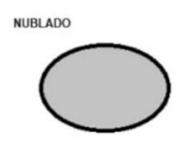

Nublado

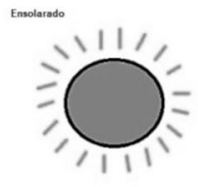

Ensolarado $\checkmark$

(

$\square$

Figura 4 - O que é tempo Meteorológico - parte 1. 


\section{$\leftarrow \quad$ ClimaCE}

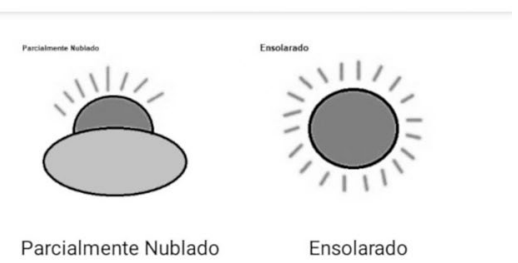

A variação do tempo meteorológico ocorre a partir da combinação de fatores como:

$$
\begin{aligned}
& \text {-> Temperatura do ar; } \\
& \text {-> Umidade relativa do ar; } \\
& \text {-> Pressão atmosférica; } \\
& \text {-> Ventos; } \\
& \text {-> Nebulosidade; }
\end{aligned}
$$

O tempo pode "mudar" de uma hora para outra, por isso a importância da previsão do tempo diária, informada nos telejornais e sites.

Você sabia? No estado do Ceará, a previsão do tempo é realizada pela FUNCEME.

\section{Maiores informações em: FUNCEME}

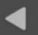

\section{$\square$}

Figura 5 - O que é tempo Meteorológico - parte 2.

\section{$\leftarrow \quad$ ClimaCE}

\section{Clima}

Para se classificar o Clima de uma cidade ou região, por exemplo, é preciso estudar a ocorrência de todos os eventos de Tempo Meteorológico que ali ocorreram, por um período de tempo de no mínimo $\mathbf{3 0}$ anos.

Alguns fatores climáticos podem influenciar em maior ou menor intensidade no clima de uma cidade ou região. Exemplos:

-> Latitude;

$\rightarrow$ Altitude;

$\rightarrow$ Maritimidade;

$\rightarrow$ Continentalidade;

$\rightarrow$ Massas de Ar;

-> Correntes Marítimas;

Por conta da distribuição desigual de calor entre as zonas térmicas da Terra, nosso planeta possui diversos tipos de clima. Para maiores informações, clique no link a seguir.

\section{4}

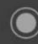

Figura 6 - O que é Clima.

sobre os principais sistemas meteorológicos responsáveis pelas precipitações durante a Pré-estação, Período Chuvoso e Pós-estação no estado do Ceará, como pode ser visualizado na Fig. 7.

Ao selecionar o nome de um sistema meteorológico disponível (Frentes Frias, VCAN, ZCIT e Ondas de Leste), outra tela do aplicativo é aberta, contendo explicações sobre o sistema meteorológico selecionado.

As Figs. 8 e 9 ilustram as informações da aba 5 Fenômenos que interferem nas chuvas no estado do Ceará, e indicam como os eventos de interação oceano-atmosfera El Niño e La Niña e o Dipolo do Atlântico podem influenciar no regime de chuvas do Semiárido nordestino.

A aba 6, intitulada "Você Sabia?", traz curiosidades sobre o estado do Ceará ligadas à Climatologia, tais como a diferença entre "inverno" e "período chuvoso", a classificação dos principais rios do Ceará e dados sobre os açudes do estado, incluindo o Castanhão, conforme pode ser visualizado na Fig. 10.

A aba 7 - Teste seus conhecimentos, representada pelas Figs. 11 e 12 contém um Quiz com dez perguntas relacionadas ao conteúdo do aplicativo.

Por fim, na aba 8, são disponibilizados links de redirecionamento para as páginas eletrônicas da FUNCEME, Instituto Nacional de Pesquisas Espaciais (INPE), Instituto Nacional de Meteorologia (INMET), entre outros, como pode ser visualizado na Fig. 13.

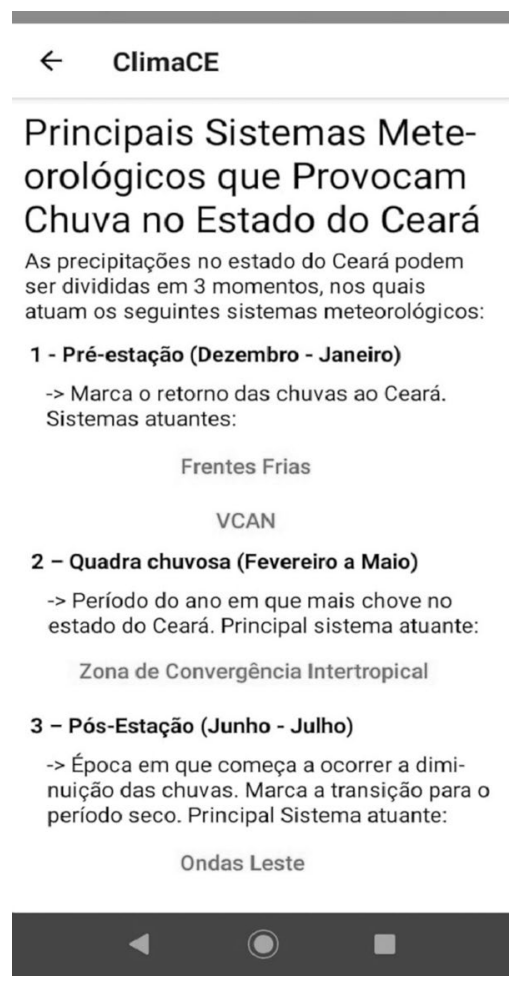

Figura 7 - Principais sistemas meteorológicos que provocam chuva no estado do Ceará. 


\section{$\leftarrow \quad$ ClimaCE}

\section{Fenômenos que Inter- ferem nas Chuvas no Estado do Ceará}

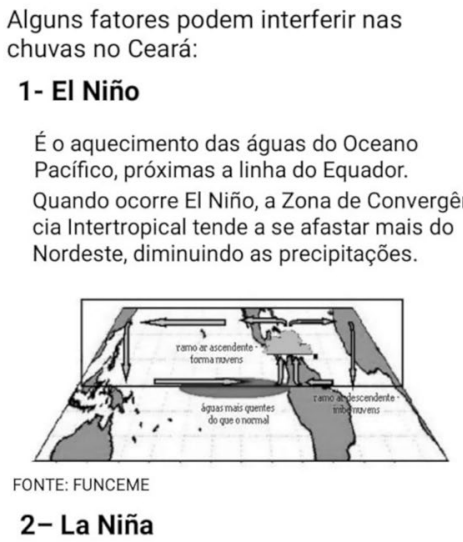

É o resfriamento das águas do Oceano Pacífico próximas a linha do Equador.

\section{$4 \quad 0$}

Figura 8 - Fenômenos que interferem nas chuvas no estado do Ceará - El Niño e La Niña.

\section{$\leftarrow \quad$ ClimaCE}

Quando ocorre La Niña, há uma tendência a que as chuvas no norte da região nordeste fiquem acima da média.

Para isso ocorrer, o oceano Atlântico precisa estar aquecido próximo ao Nordeste.

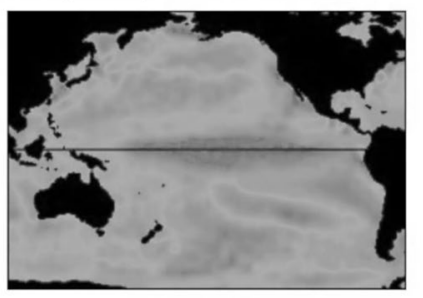

FONTE: FUNCEME

\section{3 - Dipolo do Atlântico}

As temperaturas das águas do oceano atlântico influenciam nas chuvas no norte do Nordeste.

Para maiores detalhes, visualize a tabela a seguir.

\begin{tabular}{|l|c|c|c|}
\hline Negativo & Frias & Aquecidas & Favoráveis \\
\hline Ausente & Normal & Normal & Não influencia \\
\hline
\end{tabular}

\section{$4 \quad 0$}

Figura 9 - Fenômenos que interferem nas chuvas no estado do Ceará La Niña e Dipolo do Atlântico.

\section{$\leftarrow \quad$ ClimaCE}

\section{Você Sabia?}

\section{Período chuvoso e Inverno não são a mesma coisa! \\ Período chuvoso: época do ano em que mais} chove numa cidade, estado ou região.

Inverno: é uma das quatro estações do ano. No Ceará, corresponde a uma das épocas mais secas, com início no final do mês de junho, e término em setembro.

2. A maioria dos rios do Ceará são intermitentes!

Por conta da irregularidade das chuvas, a maioria dos rios do Ceará são temporários.

O açude Castanhão é o maior reservatório do Ceará e da América Latina.

155 açudes são monitorados pela COGERH.

\section{Mais Informações}

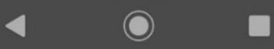

Figura 10 - Você sabia?

\section{$\leftarrow \quad$ ClimaCE}

\section{Teste seu Conhecimento}

Responda ao quiz a seguir e veja se você está por dentro da situação climática do estado do Ceará!

1) O Clima predominante no estado do Ceará é o:
A) Desértico
B) Semiárido
C) Tropical

D) Equatorial

2) Não é considerado um fator climático:

A) Latitude

B) Altitude

C) Continentalidade

D) Precipitação

3) A Pré-estação chuvosa no Ceará ocorre entre os meses de:
A) Dezembro e Janeiro
B) Fevereiro e Março
C) Abril e Maio
D) Junho e Julho

\section{4 ()}

Figura 11 - Teste seus conhecimento. 


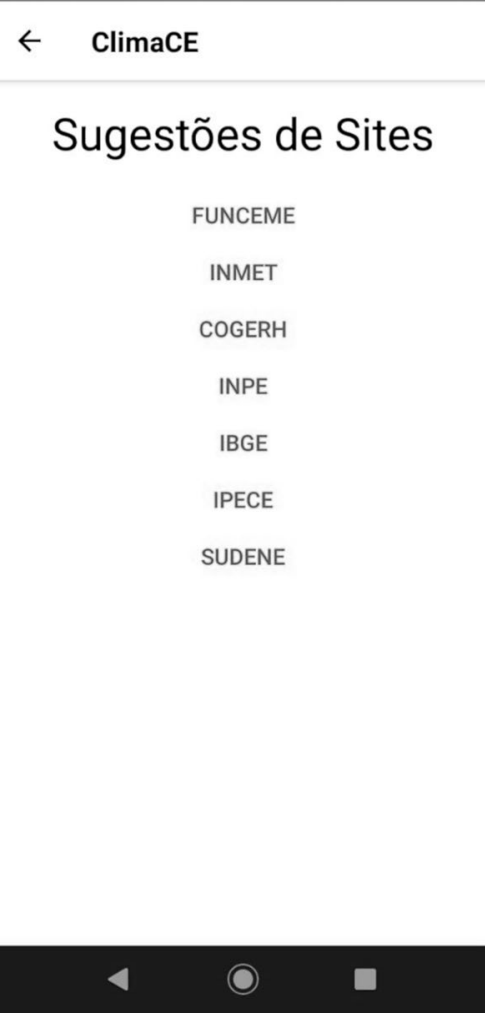

Figura 12 - Resultado do Quis.

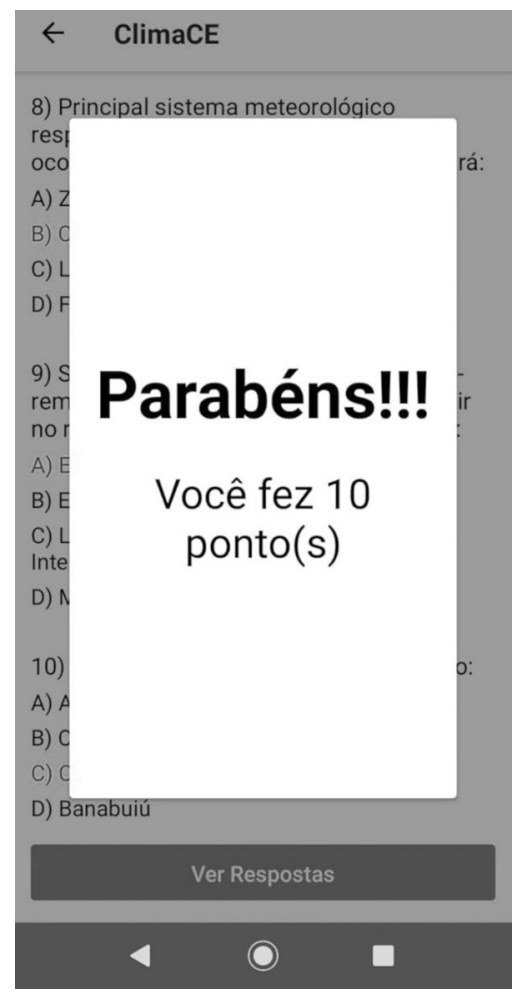

Figura 13 - Sugestões de sites.
Para a construção do aplicativo "ClimaCE" foi utilizada a linguagem JavaScript, através da estrutura React Native. Grillo e Fortes (2008, p.3) afirmam que o JavaScript é "uma linguagem de programação de propósito geral, dinâmica e possui características do paradigma de orientação a objetos. Ela é capaz de realizar virtualmente qualquer tipo de aplicação, e rodará no browser do cliente."

Sobre o React Native, Eisenman (2016, p.1) informa que é "uma estrutura JavaScript para escrever aplicativos móveis reais e nativos para iOS e Android. Ele é baseado no React, a biblioteca JavaScript do Facebook." [traduzido pelo autor]. O Sistema Gerenciador de Banco de Dados (SGBD) utilizado foi o MySQL, que "fornece uma solução de banco de dados integrada, fácil de usar para ajudar as organizações a proteger dados confidenciais contra usos não autorizados, ocultando e substituindo valores reais por subtítulos." (MySQL, 2018. Traduzido pelo autor).

O conteúdo do aplicativo "ClimaCE" foi apresentado ao grupo de vinte e seis estudantes matriculados na disciplina eletiva de "Mudanças Climáticas", da Escola de Ensino Médio em Tempo Integral - E.E.M.T.I Renato Braga, localizada em Fortaleza-CE. Na sequência, os estudantes responderam às questões do Quiz para testar os seus conhecimentos.

A disciplina citada anteriormente pertence à parte diversificada do currículo dos alunos matriculados na modalidade do Ensino Médio em Tempo Integral, podendo ser ofertada semestralmente. Foi criada com o objetivo de difundir para os alunos conceitos ligados à disciplina de Geografia e áreas afins, como a Climatologia.

Como forma de comparação dos resultados obtidos pelos alunos da eletiva, que primeiro tiveram acesso ao conteúdo do aplicativo "ClimaCE", o Quiz também foi aplicado a outros vinte e seis estudantes da E.E.M.T.I Renato Braga. A escolha ocorreu mediante sorteio realizado entre os estudantes que não estavam matriculados na eletiva e, portanto, não tinham conhecimento das informações do referido aplicativo.

\section{Resultados e Discussão}

A primeira questão do Quiz tratava sobre o clima predominante no estado do Ceará - clima Semiárido. Entre os alunos da eletiva o total de acertos foi de vinte e cinco ou $96,15 \%$. Enquanto isso, no grupo controle foram somente sete acertos, o que representa uma taxa de $26,92 \%$. Já os erros foram dezenove, totalizando um valor de $73,08 \%$.

Na segunda questão foi perguntado o que não seria considerado um fator climático. A resposta era precipitação. Na turma da disciplina eletiva, com o uso do aplicativo, a quantidade de acertos foi de dezenove, o que equivale a uma taxa de $73,08 \%$. O total de erros foi de sete, ou $26,92 \%$. 
No grupo controle, formado por estudantes que não participavam da eletiva, o número de acertos foi de catorze, o que equivale a uma taxa de $53,84 \%$. Já o total de erros foi de doze, ou $46,16 \%$, aproximadamente.

A terceira pergunta indagava sobre os meses de ocorrência da Pré-estação chuvosa no Ceará - que são os meses de Dezembro e Janeiro. Entre os integrantes da turma eletiva houve um total de vinte e quatro acertos, o equivalente a $92,31 \%$ e apenas dois erros ou $7,69 \%$.

Enquanto isso, a quantidade de acertos entre os alunos do grupo controle foi de apenas oito, ou $30,77 \%$. Nesse último grupo, o total de erros foi dezoito, ou aproximadamente $69,23 \%$.

Na quarta questão, perguntou-se o nome de um dos sistemas meteorológicos responsáveis pelas chuvas no período da Pré-estação chuvosa no Ceará. A resposta era Frentes Frias.

Observou-se que os alunos que tiveram acesso aos dados do aplicativo tiveram um melhor desempenho. Foram contabilizados dezoito acertos, o equivalente a $69,23 \%$ do total. Erros foram oito, ou $30,77 \%$ do quantitativo de estudantes.

Por sua vez, no grupo controle, o resultado foi praticamente o oposto. Houve apenas sete acertos, o que representa $26,92 \%$ do total. Ao passo que o quantitativo de erros foi de dezenove, ou 73,0\%.

A quinta questão perguntava acerca dos meses em que ocorre o período chuvoso no estado do Ceará - que vai de Fevereiro a Maio.

Entre os alunos da turma eletiva foram observados vinte e cinco acertos, ou 96,15\%. Apenas um integrante desse grupo errou a resposta, o que representa em números percentuais um total de $3,85 \%$.

Já entre os estudantes do grupo controle, o total de acertos foi dez, ou $38,46 \%$. Por sua vez, o número de erros chegou a dezesseis, o que representa em números percentuais um valor de $61,54 \%$.

A sexta questão perguntava o nome do principal sistema meteorológico responsável pelas precipitações que ocorrem no período chuvoso no estado do Ceará, que é a Zona de Convergência Intertropical - ZCIT.

Entre os estudantes da turma eletiva foram contabilizados um total de quinze acertos, o que representa em números percentuais um valor de $57,69 \%$. Erros somaram onze, ou $42,31 \%$.

Já entre os participantes do grupo controle foram contabilizados um total de sete acertos, ou $26,92 \%$. Os erros chegaram a dezenove, o que representa em números percentuais uma taxa de 73,08\%.

$\mathrm{Na}$ sétima questão perguntou-se em quais meses do ano ocorre a chamada pós-estação chuvosa. A resposta correta seriam os meses de Junho e Julho.

Foram contabilizados vinte e cinco acertos, ou $96,15 \%$ entre os participantes da turma eletiva. Apenas um erro foi detectado, ou em números percentuais, $3,85 \%$.
Já no grupo controle, foram registrados doze acertos, ou $46,15 \%$ do total. Erros somaram catorze, o equivalente a $53,85 \%$.

A oitava questão perguntou o nome do principal sistema meteorológico responsável pelas precipitações que ocorrem na Pós-estação chuvosa no estado do Ceará. A resposta correta seria Ondas de Leste.

Nessa questão, em ambos os grupos, observou-se uma quantidade de acertos inferior ao número de erros. Porém, a turma eletiva ficou à frente da turma controle, por uma pequena vantagem, com dois acertos a mais. Entre os alunos da turma eletiva, o desempenho foi satisfatório para doze estudantes, o que representa um total de $46,15 \%$. Já a taxa de erros nessa questão chegou a 53,85\%

Entre os discentes do grupo controle, os números também foram parecidos. Foram registrados dez acertos ou $38,46 \%$ do total. Erros somaram dezesseis, o que equivale a $61,54 \%$.

$\mathrm{Na}$ nona questão, perguntou-se o nome dos dois fenômenos que ocorrem no oceano Pacífico e podem interferir no regime de chuvas no estado do Ceará, que são o "El Niño" e a "La Niña.

Entre os alunos da eletiva, o total de acertos foi de vinte e quatro ou $92,30 \%$. Apenas dois estudantes erraram essa questão, o equivalente a $7,7 \%$.

No grupo controle foram registrados seis acertos, ou $23,08 \%$. Já a quantidade de erros chegou a vinte, o que equivale a uma taxa de $76,92 \%$.

A décima e última questão do Quiz perguntava aos alunos o nome do maior açude do estado do Ceará, que é o Castanhão.

Enquanto o desempenho dos alunos da turma eletiva foi de $100 \%$, no grupo controle, foram dezoito acertos, ou $69,23 \%$ e oito erros, o que equivale a uma taxa de $30,77 \%$.

A Tabela 1 a seguir mostra a análise comparativa de desempenho entre as turmas controle e eletiva, indicando a quantidade de acertos e erros, em números absolutos e percentuais, em ambos os grupos da pesquisa.

\section{Conclusões}

Os resultados dos questionários aplicados (QUIZ) indicam que houve um melhor desempenho dos alunos da eletiva, quando comparados com os da turma controle, acerca do conhecimento sobre o clima predominante, meses de ocorrência da Pré-estação, Estação chuvosa e Pós-estação, bem como dos sistemas meteorológicos responsáveis pelas precipitações sobre o estado do Ceará.

Interessante destacar que, se comparadas com as demais questões do Quiz, as de número 4, 6 e 8 foram as que registraram as menores taxas de acertos entre os alunos da eletiva, embora esse resultado ainda tenha sido superior ao observado pela turma controle.

Uma das hipóteses para isso reside na dificuldade que os alunos encontraram, mesmo entre os que utilizaram 
Tabela 1 - - Comparação de desempenho entre os alunos das turmas controle e eletiva em números absolutos e percentuais.

\begin{tabular}{|c|c|c|c|c|c|c|c|c|}
\hline \multirow[b]{3}{*}{ Questões } & \multicolumn{4}{|c|}{ Turma eletiva } & \multicolumn{4}{|c|}{ Turma controle } \\
\hline & \multicolumn{2}{|c|}{ Acertos } & \multicolumn{2}{|c|}{ Erros } & \multicolumn{2}{|c|}{ Acertos } & \multicolumn{2}{|c|}{ Erros } \\
\hline & $\mathrm{n}$ & $\%$ & $\mathrm{n}$ & $\%$ & $\mathrm{n}$ & $\%$ & $\mathrm{n}$ & $\%$ \\
\hline 1 & 25 & 96,15 & 1 & 3,85 & 7 & 26,92 & 19 & 73,08 \\
\hline 2 & 19 & 73,08 & 7 & 26,92 & 14 & 53,84 & 12 & 46,16 \\
\hline 3 & 24 & 92,31 & 2 & 7,69 & 8 & 30,77 & 18 & 69,23 \\
\hline 4 & 18 & 69,23 & 8 & 30,77 & 7 & 26,92 & 19 & 73,08 \\
\hline 5 & 25 & 96,15 & 1 & 3,85 & 10 & 38,46 & 16 & 61,54 \\
\hline 6 & 15 & 57,69 & 11 & 42,31 & 7 & 26,92 & 19 & 73,08 \\
\hline 7 & 25 & 96,15 & 1 & 3,85 & 12 & 46,15 & 14 & 53,85 \\
\hline 8 & 12 & 46,15 & 14 & 53,85 & 10 & 38,46 & 16 & 61,54 \\
\hline 9 & 24 & 92,3 & 2 & 7,7 & 6 & 23,08 & 20 & 76,92 \\
\hline 10 & 26 & 100 & 0 & 0 & 18 & 69,23 & 8 & 30,77 \\
\hline
\end{tabular}

Fonte: Elaborada pelo autor.

o aplicativo "ClimaCE", com o nome dos sistemas meteorológicos que atuam na Pré-estação, Período chuvoso e Pós-estação chuvosa no Ceará, que são respectivamente Frentes Frias, Zona de Convergência Intertropical ZCIT e Ondas de Leste.

Essa hipótese ganha consistência uma vez que as questões de números 3,5 e 7 , que as antecederam, perguntavam respectivamente, sobre os meses de ocorrência da Pré-estação, Período chuvoso e Pós-estação, e o resultado observado foi satisfatório, com índices de acertos variando entre 24 e 25 acertos, dentro de um universo de 26 alunos que responderam ao Quiz.

Isso indica que novas estratégias precisam ser pensadas para facilitar a aprendizagem do nome dos sistemas meteorológicos que causam chuvas no estado do Ceará, tais como Frentes Frias, Zona de Convergência, Ondas de Leste, entre outros.

Por outro lado, os desafios para os docentes com o Ensino Integral aumentaram, uma vez que eles, além de terem que ministrar disciplinas da Base Nacional Comum Curricular (BNCC) precisam planejar aulas eletivas sobre os mais diversos temas que sejam capazes de chamar a atenção dos alunos, o que demanda um maior tempo para planejamento dessas atividades.

Esse novo modelo de ensino proposto permite aos educadores uma maior liberdade pedagógica, o que é positivo. Cabe ao docente romper a barreira do comodismo e da zona de conforto e lançar-se em novos desafios, criando novas estratégias e metodologias capazes de transformar o ambiente da sala de aula num local prazeroso para se aprender coisas novas.

Nesse processo de mudança pelo qual a educação vem passando, é fundamental que o docente possa estar sempre atualizado diante dos novos avanços da ciência e da tecnologia, para que o processo de ensino-aprendizagem também possa evoluir.

Acredita-se que o uso do aplicativo "ClimaCE", com conceitos ligados ao clima, aos sistemas meteorológicos e aos principais sistemas atmosféricos que interferem nas precipitações estaduais possa vir a ser mais uma ferramenta disponível no processo de ensino-aprendizagem, podendo ser utilizado em aulas eletivas ou em disciplinas da BNCC nas escolas públicas e/ou particulares do estado, colaborando para o processo de aprendizagem dos alunos e da sociedade em geral sobre a Climatologia do Ceará.

\section{Referências}

BLANK, D.M.P. O contexto das mudanças climáticas e as suas vítimas. Revista Mercator, v. 14, p. 157-172, 2015.

CEARÁ. Portal Hidrológico do Ceará. Disponível em http:// funceme.br/hidro-ce-app/reservatorios/volume, acesso em 01 abr. 2020.

EISENMAN, B. Learning React Native. Ed. O'Really Media, Sebastopol, 2016.

ESTUDO do banco mundial analisa impactos do aquecimento global na seca do Nordeste brasileiro. Painel Brasileiro de Mudanças Climáticas. Disponível em http://www.pbmc. coppe.ufrj.br/pt/noticias/398-estudo-do-banco-mundial-ana lisa-impactos-do-aquecimento-global-na-seca-no-nordestebrasileiro, acesso em 10 abr. 2019.

FOLHES, M. T.; NELSON, D. Previsões tradicionais de tempo e clima no Ceará: o conhecimento popular à serviço da ciência. Revista Sociedade \& Natureza, v. 19, p. 19-31, 2007.

FUNDAÇÃO CEARENSE DE METEOROLOGIA E RECURSOS HÍDRICOS. Sistemas Meteorológicos Causadores de Chuva na Região Nordeste do Brasil. Fortaleza: FCMRH, 2005. Disponível em http://www.funceme.br/ produtos/script/chuvas/Grafico_chuvas_postos_pluvio metricos/entender/entender $\mathbf{h t m}$, acesso em 18 jan. 2019.

GRILLO, F.N.; FORTES, R.P.M. Aprendendo JavaScript. São Carlos: USP, 2008. Disponível em http://conteudo.icmc. usp.br/CMS/Arquivos/arquivos_enviados/BIBLIOTE CA_113_ND_72.pdf, acesso em 30 dez. 2018.

MYSQL. 2018. MYSQL. Disponível em https://www.mysql. com/, acesso em: 30 dez. 2018.

QUEIROZ, R. O Quinze. 98. ed. Rio de Janeiro: Editora José Olympio, 2015.

VIANELLO, R.L.; ALVES, A. Meteorologia Básica e Aplicações. UFV: Viçosa, 2000. Disponível em http://www.ebah. com.br/content/ABAAAfnhYAL/vianello-meteorologia, acesso em 23 jun. 2017.

License information: This is an open-access article distributed under the terms of the Creative Commons Attribution License (type CC-BY), which permits unrestricted use, distribution and reproduction in any medium, provided the original article is properly cited. 